Meta

Journal des tradlucteurs

Translators' Journal

\title{
Dans les coulisses de la traduction
}

\section{Henri Van Hoof}

Volume 28, numéro 4, décembre 1983

URI : https://id.erudit.org/iderudit/003084ar

DOI : https://doi.org/10.7202/003084ar

Aller au sommaire du numéro

Éditeur(s)

Les Presses de l'Université de Montréal

ISSN

0026-0452 (imprimé)

1492-1421 (numérique)

Découvrir la revue

Citer cet article

Van Hoof, H. (1983). Dans les coulisses de la traduction. Meta, 28(4), 334-343.

https://doi.org/10.7202/003084ar d'utilisation que vous pouvez consulter en ligne.

https://apropos.erudit.org/fr/usagers/politique-dutilisation/ 


\section{DANS LES COULISSES DE LA TRADUCTION}

HENRI VAN HoOF

La traduction est de tous les temps. Elle a toujours existé. Elle fait partie intégrante de la vie intellectuelle de toutes les nations. On a traduit pour découvrir une culture, un savoir. On a traduit pour répandre ou défendre des idées religieuses, philosophiques, politiques. On a traduit par patriotisme, pour lutter contre un oppresseur. On a traduit pour créer une langue nationale. On a traduit pour révéler une littérature. On a traduit par enthousiasme pour une cuvre, par admiration pour un auteur. On a traduit pour faire progresser les sciences et les techniques. On a traduit pour mille et une raisons.

Parmi toutes les motivations, il en est une dont la finalité paraît moins évidente, celle notamment qui consiste à utiliser la traduction comme un subterfuge, à faire passer pour traduction une cuvre originale. Qu'est-ce qui peut bièn pousser un auteur à pareille démarche?

\section{DE QUELQUES SUPERCHERIES}

Le phénomène n'est pas récent puisque, au Ve siècle déjà, l'Historia excidio Troiae se voulait une traduction du témoignage oculaire de Phrygius Dares, prêtre d'Héphaïstos à Troie, cité dans l'Iliade d'Homère. Au XII ${ }^{e}$ siècle, en Grande-Bretagne, Geoffrey of Monmouth (1100-1154), évêque de Saint-Asaph et historien gallois, écrivit en latin une Historia Regnum Britanniae $( \pm 1139)$ qu'il prétendait avoir traduite d'un ancien ouvrage en langue vulgaire, alors qu'il s'agissait d'une mystification pure et simple.

Même certains chefs-d'œuvre de la littérature universelle ont été présentés comme des traductions. Cervantès, par exemple, assure au début de son Don Quichotte (1605) qu'il en avait trouvé le texte, écrit en «caractères arabesques », chez un brocanteur juif de Tolède. Il aurait chargé un Morisque de le mettre en castillan «sans en rien ôter ni ajouter». Ce dernier aurait ainsi traduit en un peu moins de six semaines l'œuvre tout entière, qui se serait intitulée Histoire de don Quichotte de la Manche, écrite par Cid Hamet Benengeli, historien arabique.

À un niveau de moindre célébrité, le dramaturge Andreas Greif, dit Gryphius (1616-1664), principal représentant des poètes du haut baroque allemand, a présenté sa comédie Absurdia Comica (1658) comme l'adaptation d'une traduction de Shakespeare faite par un certain Schwenter et qu'il aurait simplement «besser ausgerüstet ». Est-ce humilité ou astuce qui incite ces auteurs à s'abriter derrière un traducteur fictif?

Parfois, les raisons sont bien plus manifestes. On comprend un Voltaire (1694-1778), qui a préféré éviter les désagréments en publiant sous ce couvert 
son roman philosophique Candide ou l'Optimisme, traduit de l'allemand de M. le Docteur Ralph, avec les additions qu'on a trouvées dans la poche du docteur lorsqu'il mourut à Minden (1759). Le docteur Ralph n'était bien entendu qu'un personnage imaginaire derrière lequel Voltaire n'eut pas tort de s'effacer, car dès le 2 mars 1759 Candide fut dénoncé au Conseil de Genève et bientôt brûlé par le bourreau.

Dans le même temps, la Grande-Bretagne était secouée par la supercherie du poète écossais James Macpherson (1736-1796), grand spécialiste du folklore des Highlands. Macpherson avait fait la connaissance de l'auteur dramatique John Home, auquel il récita quelques poésies qu'il disait avoir traduites du gaélique et qu'il attribuait à un barde nommé Ossian. Home et son ami Thomas Carlyle ramenèrent ces poèmes à Londres et les soumirent au critique littéraire Hugh Blair, qui fut enthousiasmé par l'œuvre du soi-disant Ossian et aida Macpherson à faire publier sa traduction. Elle parut en 1760 sous le titre Fragments of Ancient Poetry Collected in the Highlands and Translated from the Gaelic and Erse Language. Le succès fut tel que plusieurs écrivains écossais incitèrent Macpherson à parcourir les montagnes du Nord de l'Écosse pour y découvrir d'autres œuvres anciennes. Dans les trois années qui suivirent, il fit alors parâ̂tre deux longs poèmes épiques, Fingal (1762) et Temora (1763), qu'il prétendit avoir traduits sur des originaux gaéliques du IIIe siècle et qui lui rapportèrent une petite fortune. Mais c'est à la suite de la publication de ces livres que certains lettrés commencèrent à émettre des doutes sur leur authenticité. Samuel Johnson, le critique redouté, se déclara du même avis et dénonça les poèmes d'Ossian comme des faux dans son Journey to the Western Isles of Scotland (1775). Invité à produire les originaux, Macpherson ne put qu'exhiber une lettre de son éditeur qui certifiait les avoir eus entre les mains. La querelle s'envenima et divisa la Grande-Bretagne entre défenseurs et détracteurs de Macpherson. La plupart de ses défenseurs invoquaient l'argument que ses autres productions poétiques et sa traduction de l'Iliade d'Homère, prosaïque et plate, prouvaient sans conteste l'incapacité de Macpherson à composer les magnifiques chants sauvages incriminés. Quoi qu'il en soit, la critique moderne considère que les poèmes ont été bel et bien inventés par lui, et lorsqu'on parle du barde on pense à l'écrivain écossais dont l'influence sur la littérature romantique fut considérable.

L'imposture du pasteur Samuel Henley, commise vers la même époque, eut moins de retentissement. En 1786, il fit paraitre The History of the Caliph Vathek, prétendument traduite de l'arabe. Il s'agissait en réalité d'une traduction pirate du conte oriental Vathek écrit en français par le romancier William Beckford (1760-1844) à l'âge de vingt-deux ans, mais non édité. Pour dévoiler la supercherie, Beckford publia l'original français l'année suivante. Dans le cas de Henley, comme dans celui de Macpherson, la recherche de la gloire, fût-ce d'une gloire factice, ne semble pas étrangère à leur comportement.

Faut-il interpréter dans le même sens les cuvres par lesquelles Prosper Mérimée (1803-1870) fit ses premiers pas en littérature? Faut-il y voir vraiment un désir de réussite plus rapide ou davantage un goût naturel pour la mystification? Le fait est qu'il débuta en 1825 en publiant le Théâtre de 
Clara Gazul qui était censé avoir été écrit par une actrice espagnole et dont il se présentait comme le simple traducteur dans une préface signée Joseph L'Estrange. Certes, Mérimée était doué pour les langues et il avait appris l'espagnol comme il avait appris le grec et l'anglais, comme plus tard il apprendrait le russe. Il pouvait donc très bien passer pour le traducteur d'un tel ouvrage. Deux ans plus tard, il renouvelait sa supercherie en la déguisant à peine, puisque le titre du livre, La Guzla ou choix de poésies illyriques (1827), est tout bonnement l'anagramme de Gazul. Il se faisait passer cette fois pour le traducteur d'un écrivain illyrien, Hyacinthe Maglonovich. En réalité, il avait composé ce recueil en quinze jours, en s'appuyant sur quelques récits de voyages et en $y$ incorporant quelques mots illyriens pour faire couleur locale. Le succès de ces ballades fut immense; elles furent traduites - réellement, cette fois - en allemand et Pouchkine lui-même en mit quelques-unes en russe.

Plus claires sont les motivations d'un Willibald Alexis (1798-1871), romancier allemand d'origine française, qui crut bon de profiter de la popularité dont Walter Scott jouissait en Allemagne pour lancer ses deux premiers livres, Waldamor (1824) et Schloss Avalon (1827), en prétendant qu'ils avaient été traduits du père d'Ivanhoé. Le cas de son contemporain, le poète Friedrich von Bodenstedt (1819-1892) est plus complexe, en ce sens que le personnage dont celui-ci se disait le traducteur a vraiment existé. Professeur de philologie slave, von Bodenstedt voyagea dans le Caucase et séjourna un an à Tbilissi (en ce temps-là Tiflis), où il apprit le tartare, le georgien, le persan et l'arménien. Il y fit la connaissance d'un Persan, Mirza Schaffy, qui allait lui servir de prêtenom pour ses Lieder des Mirza Schaffy (1851), poèmes pseudo-orientaux prétendument traduits du tartare. Le mystification ne fut découverte qu'en 1874 . Elle n'empêcha pas von Bodenstedt de mériter l'estime de l'élite intellectuelle de son temps pour d'authentiques traductions de Pouchkine, de Tourgueniev et de Lermontov pour le russe, de Hafiz et d'Omar Khayyam pour le persan. Toujours dans l'Allemagne du XIX ${ }^{\mathrm{e}}$ siècle, c'est l'influence de la littérature scandinave qui semble avoir poussé les amis Arno Holz (1863-1929) et Johannes Schlaf (1862-1941) à publier ensemble, sous le pseudonyme de Bjarne P. Holmsen, trois études sur le naturalisme prétendument traduites des langues nordiques.

En France, le poète et romancier Pierre Louÿs (1870-1925) s'était déjà taillé une certaine réputation avec des traductions des Poésies de Méléagre de Gadara (1893) et des Scènes de la vie des courtisanes (1894) de Lucien. Est-ce pour cela qu'il décida de présenter ses Chansons de Bilitis (1894) comme la traduction des œuvres d'une poétesse de l'Antiquité? On peut se demander si ce chef-d'œuvre, l'un des plus beaux exemples de poèmes en prose de la langue française, avaient besoin de ce subterfuge. Plus près de nous, Boris Vian (1920-1959), autre traducteur de talent lorsqu'il s'attaquait aux pièces du dramaturge suédois August Strindberg, avait sans doute de meilleures raisons de recourir à la supercherie lorsqu'il publia sous le couvert d'une traduction le roman noir J'irai cracher sur vos tombes (1946). Attribué à un fantomatique auteur américain du nom de Vernon Sullivan, ce livre qui traite de la sexualité, de la violence et du racisme, provoqua un grand scandale. 


\section{DE QUELQUES PHÉNOMÈNES}

Si l'on a traduit pour les motifs les plus variés, les traducteurs, quant à eux, viennent aussi d'une grande variété d'horizons. Certains se distinguent par leur rang social, d'autres par l'anachronisme ou la visée futuriste de leurs entreprises, d'autres encore par l'étendue de leurs connaissances linguistiques. Leur cohorte compte même un certain nombre de têtes couronnées.

Le roi Alfred (849-901), premier souverain anglais après sa victoire sur les Danois, fut un traducteur émérite. Désireux de promouvoir l'instruction de ses sujets, il traduisit dans un but pédagogique de nombreux ouvrages latins tels l'Ecclesiastical History of England de Bède le Vénérable, la Universal History d'Orose, les Soliloquia de saint Augustin, le manuel religieux Pastoral Care de Grégoire le Grand et, surtout, On the Consolation of Philosophy de Boèce, probablement la meilleure des traductions royales. Une autre souveraine anglaise, la grande Elizabeth I (1533-1603), qui savait le latin et le grec, mais aussi le français et l'italien, a laissé des traductions du poète latin Boèce, de l'Ars Poetica d'Horace et du De Curiositate de Plutarque.

En France, le roi Louis XIII (1601-1643), fort pieux, timide et réservé, traduisit à l'âge de dix ans l'exposé des règles de conduite composé par le diacre Agapet pour l'empereur Justinien. Cette traduction, intitulée Préceptes d'Agapetus à Justinien, mis en françois par le roy très chrestien Louis XIIIe, fut publiée à Paris en 1612. L'un de ses successeurs, Louis XVI (1754-1793), alors qu'il était encore dauphin, traduisit de l'anglais les tomes I à IV de l'Histoire de la décadence et de la chute de l'Empire romain d'Edward Gibbon, qu'il publia en 1777 sous le pseudonyme de Leclerc de Sept-Chênes. Était-ce prémonition de sa propre chute?

En Suède, le roi Gustave III (1746-1792) mit en prose les deux premiers chants de la Henriade de Voltaire en 1789 et les envoya anonymement à l'Académie suédoise. Le secrétaire perpétuel Nils von Rosenstein émit au sujet de cette traduction l'avis suivant: «Elle témoigne de génie, de beaucoup de feu, d'un choix heureux de mots, mais parfois la construction ressemble trop à la construction française et s'écarte des règles ordinaires du suédois. ... Son auteur doit être de Stockholm»(!!).

Le roi Jean de Saxe (1801-1873) consacra plusieurs années à traduire de l'italien la Divina Commedia de Dante, qu'il publia en 1849 dans une version non rimée sous le pseudonyme de Philateles.

Plus près de nous, des chefs d'État contemporains ont poursuivi la tradition. Zalman Shazar, président d'Israël, a traduit en hébreu un recueil de poèmes du roi Mahendras du Népal. Le président Nyerere de Tanzanie a mis en swahili la tragédie Julius Caesar de Shakespeare, dont la première eut lieu à Dar-es-Salam en 1962. Le président indien Zakir Husain a traduit l'État de Platon en urdu, l'une des langues nationales de l'Inde.

Faut-il parler de phénomènes ou plutôt de rêveurs impénitents lorsqu'il s'agit de personnages qui s'astreignent à traduire encore en latin auX XIX ${ }^{e}$ et $\mathrm{XX}^{\mathrm{e}}$ siècles? Comme cet orientaliste allemand, Heinrich Fleischer (1801-1888), qui publie en 1831 Ismaelis Abulfedae historia ante-islamica, texte arabe avec 
traduction latine; comme cet autre philologue allemand Flügel, qui fait paraître de 1835 à 1858 une traduction latine du Dictionnaire bibliographique de l'érudit turc Hâdji Khalfa. Comme, beaucoup plus récemment, et pour des auvres contemporaines cette fois, Alexander Leonhard, professeur d'origine hongroise installé à Sao Paulo, au Brésil, qui met en latin le classique pour enfants Poo the Bear d'Alan Milne, mais aussi le roman Bonjour Tristesse (1963) de Françoise Sagan. Comme, encore, le Suisse Peter Wiesmann qui publie en 1965 une version latine des meilleurs poèmes de Christian Morgenstern sous le titre Carmina Lunovilia-Das Mondschaf.

Peut-on imaginer que ces traducteurs croyaient toujours en un renouveau du latin comme langue universelle, concurrençant en cela les tentatives des promoteurs de l'espéranto? Car nombreuses déjà sont les traductions parues dans cette langue artificielle. Le médecin et poète hongrois Kálmán Kalocsay (1891-1976), qui en était un chaud partisan, a traduit pour sa part Jean Vaillant du poète Sandor Petöfi, la Tragédie de l'Homme d'Imre Madach et une anthologie de poésie hongroise. En Pologne, un Kazimierz Bein a traduit le Pharaon, roman de son compatriote Boleslaw Prus, tandis qu'un Antoni Grabowski publiait sous le titre $D u$ Parnasse des peuples une anthologie de cent poèmes en trente langues, parmi lesquels Pan Tadeusz d'Adam Mickiewicz, En Suisse de Juliusz Slowacki, etc. En U.R.S.S., Nekrasov a mis en espéranto Eugène Onéguine de Pouchkine, V. Devjatnine les poèmes Poltava, Ruslan et Ludmilla et Boris Godounov du même Pouchkine. En France, Warenghien a traduit les Fleurs du Mal de Baudelaire et les Rubbaiyat d'Omar Khayyam. En GrandeBretagne, L.N.M. Newell a transposé Hamlet. Tous ces efforts, au fond, procèdent d'une motivation nouvelle et paradoxale au plan de la traduction: aider à implanter une langue conventionnelle internationale.

On conçoit que telle n'ait pas été la préoccupation de ces polyglottes du monde entier qui se sont mis en évidence par le nombre même des langues qu'ils maniaient. En France, l'écrivain Armand Robin (1912-1961), après n'avoir parlé que le breton jusqu'à l'âge de six ans, apprit le français, le grec, le latin, l'anglais, l'allemand, le russe, l'italien et l'espagnol tout en terminant son agrégation de lettres; il y ajouta le néerlandais, le finnois, le hongrois; puis, il s'inscrivit à l'École des langues orientales et se familiarisa avec le chinois et l'arabe. Un bagage de quatorze langues au total, qui lui permettra de donner des traductions de Maïakovski, Pasternak, Blok, Essenine dans Quatre poètes russes (1949), de Poèmes (1951) du Hongrois Endre Ady, de trois pièces de Goethe et de poésies de Rilke, d'auteurs chinois et arabes (du VIe s.) dans Poèmes non traduits (2 vol., 1953, 1958), de poèmes de Poe pour l'anglais, d'Ungaretti pour l'italien, d'auteurs suédois, néerlandais, etc.

L'Allemagne aussi connut quelques polyglottes de dimension. L'orientaliste Job Ludolf (1624-1704), spécialiste de l'éthiopien auquel on doit un Lexicon aethiopico-latinum (1698), passait pour savoir vingt-cinq langues. Le philologue Johann Adelung (1732-1806) est l'auteur d'un ouvrage intitulé Mithridate ou Tableau universel des langues, avec le Pater en cinq cents idiomes, dont il ne put achever que la partie consacrée aux langues asiatiques. Mais sans doute n'a-t-il fait que rassembler des traductions existant dans ces différentes langues. 
La même réserve vaut pour le Paternoster en six cent-huit langues (1844) et le Paternoster en deux cents langues (1847) de l'Autrichien Aloïs Auer (1813-1869). Wilhelm von Humboldt (1767-1835), l'un des pères de la linguistique moderne et traducteur d'Homère, de Pindare et d'Eschyle, savait, outre le grec, le français, le basque, le chinois, le kawi, dialecte de l'île de Java, etc. L'orientaliste Friedrich Rückert (1788-1866), connaissant déjà le grec et le latin, avait étudié les langues et les littératures arabe, turque et persane avec von HammerPurgstall à Vienne, pour s'intéresser ensuite à l'hébreu, au sanskrit, aux dialectes indiens, au syriaque, au copte, au tartare, au finnois. De cette palette d'une douzaine de langues sont nées des traductions du latin comme Das Leben der Hadumod (1845) du moine Agius, du grec comme Die Vögel (1832) d'Aristophane et 20 Idyllen (1858) de Théocrite, du persan comme Oestliche Rosen (1822) de Hafiz, Rostem und Suhrab (1838) de Firdousi, de l'arabe comme Die Makamen des Harari (1826-1837), Amrilkais, der Dichter und König (1843), Hamasa (1846) d'Abu Temman, anthologie de 750 poètes anciens à laquelle il travailla vingt ans, du sanskrit comme Nal und Damajanti (1826), Sanskritische Liebesliedchen aus Amaru-Satakam (1830), Sakuntala (1854-1855) de Kâlidâsa, ainsi que des extraits du Coran (1820), de l'hébreu comme Hebräische Propheten (1826-1841), etc.

En Grande-Bretagne, l'orientaliste William Jones (1746-1794), pionnier de l'étude du sanskrit et fondateur de la Bengal Asiatic Society, était très doué pour les langues. Il en apprit vingt-huit, qui lui permirent de donner des traductions du persan comme la biographie du roi Nadir Shah, Tarikh i Nadri (1770), du sanskrit comme Sakountala (1789) de Kâlidâsa, Histopadesa et bien d'autres. Un autre orientaliste, John Leyden (1775-1811), qui était aussi médecin et grand voyageur (Inde, Malaisie, Indonésie), connaissait plus de trente idiomes. Il traduisit les Évangiles en cinq dialectes indiens, ainsi que des ouvrages comme Malay Annals (1821), Commentaries of Baber (1826), etc. Le linguiste John Bowring (1792-1872) possédait un don des langues qui lui permit d'en apprendre deux cents et d'en parler cent. Son œuvre principale consiste en la traduction des chants populaires de la plupart des nations d'Europe, qu'il réunit dans des volumes comme Ancient Poetry and Romances of Spain, etc. Ses traductions de poésie néerlandaise lui valurent un doctorat de l'Université de Groningue. Il traduisit aussi du polonais dans Specimens of the Polish Poets (1827) et du français le Traité de législation civile et pénale (1802) et la Théorie des peines et récompenses (1811) écrits dans cette langue par le philosophe anglais Jeremy Bentham. L'explorateur Richard Burton (1821-1890), au cours d'une vie de voyages et d'aventures, acquit la connaissance de trente-cinq langues. Sur la fin de son existence, il en usa pour traduire The Lusiads (1881) du poète portugais Camoëns, Arabian Nights (1885-1888) dans une version littérale et voluptueuse dont sa femme publia une édition expurgée, The Pentameron (1893) du conteur napolitain Giambattista Basile. Et peut-être conviendrait-il de ranger parmi ces phénomènes la poétesse Elizabeth Barrett-Browning (1806-1861) qui lisait Homère dans le texte à huit ans, pour donner plus tard une traduction de Prometheus Bound (1833) d'Eschyle, ainsi que le prélat et historien Connop Thirlwall (1797-1875) qui lisait le latin et le grec à quatre ans avant de devenir plus tard le traducteur d'ouvrages allemands comme Critical Essay on the Gospel 
of St. Luke (1825) du théologien Friedrich Schleiermacher et The History of Rome (1828) de l'historien Barthold Niebuhr.

Il ne faudrait toutefois pas s'imaginer que ces champions du polyglottisme sont l'apanage de quelques pays occidentaux. En Roumanie, l'écrivain Dimitrie Kantemir (1673-1723), prince de Moldavie, fut l'un des hommes les plus cultivés de son époque. Destitué quelques semaines après avoir succédé à son père, il s'était rendu à Constantinople où il était entré en contact avec la culture byzantine et s'était mis à l'étude des langues. Sachant déjà le roumain, le latin et le grec ancien, il y ajouta le grec moderne, le français, l'italien, le russe, l'arabe, le persan, etc., pour atteindre finalement le total appréciable de quatorze idiomes. Il est l'auteur d'une traduction du Coran, réalisée partie en latin, partie en russe. En Yougoslavie, l'écrivain, pédagogue et homme d'État Dositej Obradovic (1740-1811), qui fut aussi un voyageur infatigable et un éternel étudiant, connaissait sur la fin de sa vie, outre le serbe, sa langue maternelle, le latin, le grec, l'albanais, le roumain, le français, l'italien, l'anglais, l'espagnol, l'allemand et le russe. Il a traduit de l'allemand les Fables (1788) et le drame Damon (1793) de Lessing, de l'italien les Novelle morali de Francesco Soave dans une version très libre intitulée Etika (1803). En Arménie, l'écrivain Hohvannes Deroyentz (1801-1888), de son vrai nom Tcharmoudjian, après avoir étudié la philosophie et la théologie, apprit seul un grand nombre de sciences et de langues, parmi lesquelles le latin, le grec, le français, l'italien, l'anglais, l'allemand, le turc, le géorgien, l'hébreu, l'arabe et le persan, devenant ainsi l'homme le plus érudit de la nation arménienne. Il a laissé diverses traductions, dont une des Pensées de Pascal. En Russie, la poétesse Elisabeth Kulmann (1808-1825) savait onze langues à l'âge de quinze ans et composait en russe, en français et en allemand. Elle a traduit du grec des Odes d'Anacréon et de Pindare, du latin des poésies d'Horace, du portugais des œuvres de Manoel. Plus remarquable encore est l'écrivain ukrainien Mikola Lukasch (1919-) qui, après avoir fait des études d'histoire à Kiev, s'inscrivit à l'Institut pédagogique des langues étrangères de Kharkov, où il découvrit sa vocation de traducteur en 1947. Il est vrai qu'il traduisait déjà des poésies de Heine et des passages du Faust de Goethe à quatorze ans. En 1955 parut sa version intégrale du Faust, une réussite magistrale. Traducteur de prose, de poésie et de théâtre au départ de vingt-deux langues - latin. russe, biélorusse, yiddish, polonais, allemand, anglais, français, italien, espagnol, hongrois, tchèque, slovaque, serbo-croate, slovène, bulgare, portugais, norvégien, danois, etc. - il a donné du français des poésies de Baudelaire et de Verlaine, Madame Bovary de Flaubert, de l'italien le Décaméron de Boccace, de l'espagnol Don Quichotte de Cervantès, des pièces de Lope de Vega et des poésies de Garcia Lorca, de l'allemand des poèmes de Schiller, du hongrois la Tragédie de l'Homme d'Imre Madach, du polonais des poésies de Mickiewicz, du norvégien Peer Gynt d'Ibsen, etc., toutes traductions qui font honneur à la langue ukrainienne. Son compatriote, le poète Pavlo Hrabovski (1864-1902) se mit à l'étude des langues en prison. Arrêté de nombreuses fois pour ses idées révolutionnaires dès l'âge de dix-huit ans, et mort en déportation d'ailleurs, c'est au cours de ses détentions répétées qu'il entreprit de traduire des poètes russes, bulgares, serbes, croates, 
slovaques, tchèques, polonais, hongrois, géorgiens, arméniens, etc. et, ensuite, allemands, anglais et français. Son volume Fate (1897) contient cent-six poèmes traduits de Poe, Longfellow, Burns, Scott, Wordsworth, Shelley, Tennyson pour l'anglais, de Baudelaire et Verlaine pour le français, de Goethe et Lenau pour l'allemand, ainsi que des auteurs danois, norvégiens, polonais, hongrois, etc. Il a traduit aussi le Faust de Goethe, The Prisoner of Chillon de Byron, The Song of the Shirt de Thomas Hood, Eugène Onéguine de Pouchkine et un recueil du poète Ivan Sourikov. Son dernier recueil, Wave (1899), venant après le volume Lute (1898), comprend cent trente-huit poèmes de 88 auteurs, mais ne parut que cinquante ans après sa mort et toujours sous le contrôle de la police.

\section{DE QUELQUES MARTYRS}

À l'inverse de Hrabovski, qui vint à la traduction alors qu'il était emprisonné pour ses idées, de nombreux traducteurs ont eu à souffrir dans leur chair ou dans leur cœur en raison même de leur activité traduisante. En France, le théologien et moraliste Jacques Lefèvre d'Étaples (1450-1537), inspirateur de la pré-Réforme française, eut la douleur de voir condamner et brûler sa traduction du Nouveau Testament (1523) qu'il avait faite directement du grec. Vers la même époque, le poète Clément Marot (1495-1544) traduisit du latin, peut-être à la requête de Marguerite de Navarre, Trente psaumes de David mis en rime françoise (1539). Accueillie avec faveur par la Cour et les Protestants, cette traduction fut censurée par la Sorbonne, qui reprochait à Marot d'avoir affadi le texte biblique. En 1542 , un mandat d'arrêt fut lancé contre le poète, qui préféra fuir la persécution en se réfugiant à Genève, puis à Turin, où il mourut peu de temps après. L'humaniste Étienne Dolet (1509-1546) fut moins heureux. Ce philologue et imprimeur, l'un des plus énergiques représentants de la renaissance intellectuelle en France, s'était hissé au premier rang des érudits par ses ouvrages sur la langue latine et par ses traductions du grec et du latin. L'étude de l'Antiquité avait développé chez lui l'esprit d'examen et il s'était lancé avec ardeur dans le mouvement favorable aux idées nouvelles. Accusé d'athéisme et d'hérésie, condamné à mort une première fois à Lyon (1542), où il avait son imprimerie, pour avoir publié en français le Nouveau Testament, les Épîtres et Évangiles et les Psaumes, il obtint des lettres de rémission de François Ier. En 1544, sous prétexte d'avoir traduit l'Antiochus de Platon, dialogue dans lequel on trouvait une négation de l'immortalité de l'âme, il fut dénoncé par la Sorbonne, condamné, pendu puis brûlé à Paris, place Maubert, où l'on voit aujourd'hui sa statue. Premier martyr de la traduction en France, Dolet fut aussi le premier à formuler des règles en matière de traduction dans un opuscule intitulé la Manière de bien traduire d'une langue en aultre (1540). Le théologien Pasquier Quesnel (1634-1719), s'il réussit à échapper au bûcher, n'en eut pas moins à souffrir une constante persécution dès qu'il eut publié sa traduction de la Vulgate sous le titre le Nouveau Testament en français avec des réflexions morales sur chaque verset (1671, remanié en 1699). Obligé de quitter la France, il se rendit à Bruxelles, où il fut néanmoins arrêté à l'instigation de l'archevêque de Malines et incarcéré dans cette ville en 1696 . Il ne sortit de captivité qu'en 1703, délivré par l'un de ses partisans. Peu après, c'est Louix XIV qui donna 
l'ordre de le reprendre, mais Quesnel put se réfugier en Hollande. En 1708, le pape Clément XI, tout dévoué au roi de France, ordonna de brûler solennellement l'ouvrage, mais la sentence ne fut pas exécutée. Alors, Louis XIV exigea une bulle condamnant Quesnel et l'obtint en 1713. Exclu de l'Église, Quesnel demeura en Hollande et y fonda de nombreuses communautés jansénistes.

La Grande-Bretagne aussi eut son martyr de la traduction. Et pour des raisons également religieuses. Le réformateur William Tyndale (1484-1536) avait déjà traduit le Manual of the Christian Soldier (1522) d'Érasme lorsqu'il forma le projet de mettre en anglais les Saintes Écritures. Il se mit à l'ouvrage sous la protection d'un riche drapier londonien, Humphrey Monmouth. Craignant toutefois pour sa personne, il partit pour Hambourg en 1524, rendit visite à Luther, commença la publication de sa traduction à Cologne, mais dut fuir à Worms pour pouvoir achever son œuvre. Le Newe Testament y parut en 1525 . De là, elle fut introduite en Angleterre, où elle fut interdite en 1528. Tyndale se retira à Marbourg, où il publia, en 1530, sa traduction du Pentateuch et, en 1531, celle du Boke of Jonas. L'hostilité du roi Henry VIII s'étant un peu calmée, il s'installa à Anvers pour réviser ses traductions. Il fut trahi et livré aux officiers impériaux qui le conduisirent à Vilvorde, près de Bruxelles, où il fut étranglé et brûlé sur le bûcher en 1536. Ses dernières paroles furent: «Lord, open the King of England's eyes».

En Espagne également, c'est la traduction religieuse qui valut des ennuis à quelques auteurs, tant religieux que laïques. L'Inquisition s'y montrait aussi intolérante que la Sorbonne en France à la même époque, parfois avec la complicité des souverains. Le cas de Franciso Enzina (1520-1552) est, de ce point de vue, édifiant. Ce théologien luthérien, connu aussi sous le nom grec de Dryander, français de Du Chêne et flamand de Van Eyck, avait traduit en espagnol le Nouveau Testament (1543), qu'il dédia à Charles-Quint. L'empereur accepta la dédicace, mais dénonça ensuite le traducteur à l'Inquisition à l'instigation de son confesseur! Le poète et prosateur Luis de Leon (1527-1591) eut, lui aussi, maille à partir avec l'Inquisition pour sa traduction splendide du chant de Salomon Cantar de cantares (1572).

En Italie, mais beaucoup plus récemment, ce sont encore des facteurs confessionnels qui ont joué un rôle. Ainsi, l'écrivain Ferdinando Ranalli (18131894), professeur d'histoire et de littérature, fut chassé de Rome en 1836 pour avoir traduit deux épitres latines de Pétrarque contre la Curie.

De nos jours, certes, on ne livre plus les traducteurs ou leurs ouvres au bourreau, mais les avanies ne leur sont pas épargnées pour autant, même si les raisons qui font agir leurs persécuteurs sont devenues plus politiques et idéologiques que religieuses. Du moins dans certains pays. C'est ainsi que la traduction grecque du Petit livre rouge de l'écolier, ouvrage danois dû à la collaboration d'un psychologue, d'un sociologue et d'un professeur, a été condamné il y a quelques années par un tribunal d'Athènes pour «incitation au crime». En Turquie, les juges semblent avoir fait du traducteur une cible privilégiée. En 1966, deux écrivains turcs qui avaient traduit des extraits des lettres de François-Émile Babeuf (1760-1797), le Gracchus de la Révolution française, ont 
été inculpés de propagande communiste et le procureur a requis contre eux une peine de sept à quinze ans de prison. En 1968, le poète Attila Hilan a été traduit en justice pour avoir publié une version turque de l'Espoir d'André Malraux, tandis que le romancier Osman Tokatli, coupable d'avoir traduit du russe le roman Ciment de Fedor Gladkov, était condamné à quinze mois de prison. En 1972, un tribunal militaire a prononcé une peine de sept ans et six mois de privation de liberté à l'encontre de l'écrivain Nasih Nuri Ileri pour avoir traduit un ouvrage sur la vie et l'œuvre de Lénine. La même année, à Ankara, le traducteur Abdullah Nefes a été condamné à un emprisonnement d'égale durée pour avoir mis de l'anglais en turc un choix des œuvres de Mao Tsé-Tung.

Que conclure de toutes ces poursuites, de toutes ces condamnations, sinon qu'elles ne sont que le reflet d'une reconnaissance implicite de la puissance de la traduction comme instrument de diffusion des idées? 